ONLINE MUTATION REPORT

\title{
Functional analysis of novel SLC1 1A1 (NRAMP1) promoter variants in susceptibility to HIV-1
}

\author{
H Donninger, T J Cashmore, T Scriba, D C Petersen, E Janse van Rensburg, V M Hayes
}

J Med Genet 2004;41:e49 (http://www.jmedgenet.com/cgi/content/full/41/4/e49). doi: 10.1136/jmg.2003.013318

$\mathrm{T}$ he divalent cation transporter is the natural resistance associated macrophage protein 1 (formerly NRAMPI and now named SLCIIAI) for solute carrier IIAl (OMIM accession number 600266). The gene that codes for this transporter has been studied intensively for its role in conferring susceptibility to infectious diseases such as tuberculosis, leprosy, meningococcal meningitis, visceral leishmaniasis, and HIV infection, as well as to autoimmune diseases such as rheumatoid arthritis, diabetes, sarcoidosis, inflammatory bowel disease, ${ }^{1}$ and, more recently, Kawasaki disease. ${ }^{2}$ Most studies have investigated a functional GT repeat sequence in the promoter region of this gene ${ }^{3}$ and have identified two commonly occurring repeat alleles and four rare repeat alleles. ${ }^{45}$ The common alleles are $\mathrm{T}(\mathrm{GT})_{5^{-}}$ $\mathrm{AC}(\mathrm{GT})_{5} \mathrm{AC}(\mathrm{GT})_{10} \quad($ allele 2$)$ and $\mathrm{T}(\mathrm{GT})_{5} \mathrm{AC}(\mathrm{GT})_{5} \mathrm{AC}(\mathrm{GT})_{9}$ (allele 3; GenBank accession number AF229163, 5768 to 5808). Allele 2, which decreases gene expression, has been associated with susceptibility to infectious diseases; the more common allele 3 enhances gene expression to protect against infectious diseases while enhancing susceptibility to autoimmune diseases. Although HIV is classified as an infectious disease, it affects the autoimmune system, which may explain why allele 3 is associated with susceptibility to HIV- $1 .{ }^{67}$ This study aimed to screen the promoter region of SLC11Al for novel sequence variations in people from subSaharan Africa infected with HIV-1 compared with uninfected people and to determine the effect of novel variants on normal promoter function.

\section{MATERIALS AND METHODS \\ Participants}

We studied 84 HIV-1 seropositive people (60 African and 24 of mixed African-European descent) and 133 HIV-1 seronegative people (64 African, 62 of mixed African-European descent, and seven Asian) who lived in the Western Cape of South Africa and who attended one of the HIV-l clinics in Tygerberg or Langa or the blood transfusion services of the Western Cape. In this study, we use "African" to define people of predominantly Xhosa descent, while the mixed African-European population (known as "Coloured" in South Africa) consists of a well defined population with origins from an initial population mixture about 300 years ago. This study population is defined in a recent publication. ${ }^{8}$ The ethics review committee of the University of Stellenbosch gave ethical approval, and informed consent was obtained from all study participants. We took blood from the patients and volunteers, and we extracted DNA with the Qiagen Extraction Kit (Qiagen, Valencia, CA, USA), as per the manufacturer's instructions.

\section{Mutation detection}

Two sets of denaturing gradient gel electrophoresis primers were designed to cover the upstream promoter region from the GT repeat sequence (from nucleotide -715 to nucleotide -488 from translation start site) and downstream promoter

\section{Key points}

- In contrast with previous studies, this study showed that polymorphic GT repeats in the promoter region of the SLC1 1A1 (NRAMP1) gene were not associated with altered risk for HIV-1 infection in African and AfricanEuropean people from sub-Saharan Africa.

- This study aimed to identify new mutations in the promoter region of this gene that might be associated with susceptibility to infection with HIV-1 within African based populations.

- Denaturing gradient gel electrophoresis was used to assay the upstream and downstream regions of the promoter GT repeat and to screen for variants in 84 HIV-1 seropositive and 133 HIV-1 seronegative people.

- Three novel mutations and a previously reported single nucleotide polymorphism (g.332C > T) were identified. No significant associations were made between the single nucleotide polymorphism and susceptibility to HIV-1, but one of the novel mutations (g.43G $>$ C) occurred in two HIV-1 seropositive people of African descent.

- Gene expression studies showed that the g.43G>C and $\mathrm{g} .75 \mathrm{~T}>\mathrm{C}$ variants enhanced promoter activity by 1.4-fold and 1.6-fold, respectively. The promoter single nucleotide polymorphism also enhanced activity 1.6-fold, but the g.561G $>$ A variant had no effect on promoter activity.

region (from nucleotide -415 to nucleotide -132 ). The upstream primer pairs were forward 5'-AACAACTCTGA GAAGGGACA-3' and reverse 5'-TCTTTGATCTGGAGTT CCAA- $3^{\prime}$, and the downstream primers were forward 5'-GGGTGTGGTCATGGGGTATT and reverse 5'-TGCCCTGCC TCTTACATCAA- $3^{\prime}$. Both forward primers were designed with a 40 base pair (bp) GC-clamp (CGCCCGCCGCGCCCC GCGCCCGGCCCGCCGCCCCCGCCCG) attached to the 5 '-end to prevent total strand dissociation during electrophoresis through a denaturing gradient. A 7 bp (GCCGCCG) GC stretch and a 10 bp (GCCGCCGCCG) GC stretch were added to the $5^{\prime}$-end of the upstream and downstream reverse primers, respectively; the latter created a single melting domain for optimal mutation detection, as previously described. ${ }^{9}$ The repeat allele configuration was determined by direct sequencing of the amplified product created with forward primer 5'-AAGACTCGCATTAGGCCAAC-3' and reverse primer 5'-GCCTCCCAAGTTAGCTCTGA-3'

We performed DNA amplification in a $50 \mu \mathrm{l}$ reaction mixture that contained $100 \mathrm{ng}$ genomic DNA, $0.1 \mathrm{mmol} / \mathrm{l}$ of each deoxyribonucleoside triphosphate, $20 \mathrm{pmol}$ of each 
primer, $2.5 \mathrm{mmol} / \mathrm{l} 10 \times$ magnesium ion reaction buffer, and 0.5 units DNA Taq polymerase (Boehringer Mannheim, Mannheim, Germany). We performed amplification under the following conditions: initial denaturation at $96^{\circ} \mathrm{C}$ for 3 minutes, 35 cycles of denaturation at $96^{\circ} \mathrm{C}$ for 45 seconds, annealing at $60^{\circ} \mathrm{C}$ for upstream amplicon and repeat region or $65^{\circ} \mathrm{C}$ for downstream amplicon for 1 minute, and elongation at $72{ }^{\circ} \mathrm{C}$ for 1 minute 15 seconds. After cycling, we performed an additional elongation step for 10 minutes, at $72^{\circ} \mathrm{C}$, followed by denaturation at $96^{\circ} \mathrm{C}$ for 10 minutes, and heteroduplexing for 45 minutes at the optimal annealing temperatures (the latter two steps were applicable only for the denaturing gradient gel electrophoresis primers). We electrophoresed upstream and downstream amplicons through a $9 \%$ polyacrylamide gel that contained a $40-80 \%$ urea and formamide denaturing gradient (100\% urea and formamide contained $7 \mathrm{M}$ urea and $40 \%$ deionised formamide) at $59^{\circ} \mathrm{C}$ and $110 \mathrm{~V}$ overnight (phorU2 system; Ingeny, Leiden, Netherlands). We subjected the repeat region amplicon and samples that showed novel denaturing gradient gel electrophoresis banding patterns to automated sequencing after product purification with a high purity polymerase chain reaction (PCR) product purification kit (Roche Diagnostics, Mannheim, Germany).

We determined allele frequencies by allele counting. We used Fisher's exact test, which included calculation of odds ratios by InStat software, to assess significance of association between HIV-l status (seropositive $v$ seronegative) and genotype for the previously reported repeat alleles, as well as the g.332C $>\mathrm{T}$ (GenBank accession number AY363243) promoter single nucleotide polymorphism. ${ }^{10}$

\section{Gene expression studies}

We analysed variant sequences with MatInspector (version 2.2; German Research Center for Biotechnology, Braunschweig, Germany) to establish possible loss or gain of transcription factor binding sites to determine the effect of novel promoter variants on promoter function (fig 1). To determine whether these novel mutations had any true effect on promoter activity, we cloned the various mutant promoters, together with the wild type promoter, upstream of the luciferase gene, and we transiently transfected the resulting constructs into 293 cells (ATCC: CRL-1573). We measured transactivation activity relative to Renilla luciferase in three independent experiments and determined means and standard deviations. All clones were sequenced to confirm the configuration of the repeat allele and promoter single nucleotide polymorphism.

\section{RESULTS}

Analysis of the 217 participants identified three of the previously reported repeat alleles (alleles 1, 2, and 3) and four single nucleotide variants (the previously reported g.332C $>\mathrm{T}$ polymorphism and three novel rare variants). All poly-

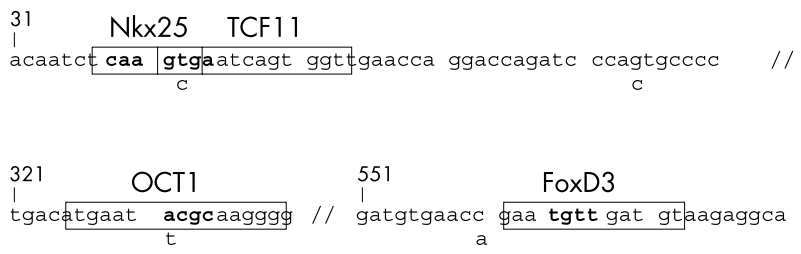

Figure 1 Schematic representation of the SLC11A1 promoter region (Genbank accession number AY363243), depicting the position of the novel mutations and known single nucleotide polymorphisms (shown below the sequence) and the various affected transcription factor binding sites (boxed regions). The core binding site for each transcription factor is highlighted in bold. morphic alleles were in Hardy-Weinberg equilibrium in the HIV seropositive and seronegative participants for both populations. The repeat allele 3 was most common in the African and African-European admixed populations, with allele frequencies of $0.84(101 / 120)$ and 0.73 (35/48) in participants infected with HIV and 0.81 (103/128) and 0.80 (99/124) in uninfected participants, respectively. We saw no significant associations between the presence of allele 3 in infected compared with uninfected African participants ( $\mathrm{p}=0.5073$, odds ratio 1.290$)$ and the mixed AfricanEuropean population $(\mathrm{p}=0.4124$; odds ratio 0.6799$)$ or in the homozygous states (Africans: $\mathrm{p}=0.3406$; AfricanEuropean: $p=0.4715$ ) or heterozygous states (African: $\mathrm{p}=0.2426$; African-European: $\mathrm{p}=0.8079$ ). Allele 2, previously associated with protection from HIV-1 infection in the homozygous state, ${ }^{6}$ was not associated with altered risk of HIV infection in our study. Three of the seven "slow progressors" (three African and four African-European participants who were asymptomatic 10 years after becoming infected with HIV-l and had not taken antiretroviral treatment) were heterozygous for allele 2, while all five "fast progressors" (three African and two African-European participants who progressed to AIDS within five years of infection with HIV-1) were homozygous for allele 3. Of the three slow progressors who were heterozygous for allele 2 (all African-European admixed), one was heterozygous for the CCR $5-\triangle 32$ bp deletion, which has been shown to be associated with slower progression of disease. Allele frequencies for the g.332C $>$ T single nucleotide polymorphism in the African and African-European admixed populations infected with HIV-1 were 0.075 (9/120) and 0.02 (1/48), respectively; the frequencies for the uninfected participants were 0.05 (6/128) and 0.072 (9/124), respectively. The promoter single nucleotide polymorphism was associated with repeat allele 3 in both populations. We saw no significant associations between HIV-l infected and non-infected African participants ( $p=0.4136$; odds ratio 1.706) and admixed African-European participants $(\mathrm{p}=0.2560$, odds ratio $0.2560)$.

The three novel sequence variants included a $\mathrm{G}$ to $\mathrm{A}$ base substitution at position 561 (g.561G $>$ A), a T to C substitution at position $75(\mathrm{~g} .75 \mathrm{~T}>\mathrm{C})$, and a $\mathrm{G}$ to $\mathrm{C}$ transition at position 43 (g.43G $>$ C) (GenBank accession number AY363243) at $-156,-642$, and -674 nucleotides from the initiator codon, respectively. The first two mutations were identified in HIV-1 seronegative participants of African and Asian descent, respectively; the g.43G $>$ C mutation occurred in two unrelated HIV-l seropositive patients of African descent. Promoter analysis showed that the $\mathrm{G}$ to $\mathrm{C}$ mutation at position 43 created a TCF1l transcription factor binding site. This mutation also interfered with a Nkx2-5 binding site, although it did not disrupt the site entirely. The single nucleotide polymorphism at position 332 introduced an OCT-1 binding site, and the transition at 561 altered, but did not destroy, a FoxD3 binding site. The 75 mutation did not create or alter any transcription factor binding sites.

We cloned these variants in a luciferase reporter system to determine their effect on promoter activity. Sequencing of the clones showed that all had the allele 3 repeat configuration and that all contained the $\mathrm{C}$ allele configuration for the g.332C $>$ T polymorphism. Promoter analysis showed that the g.43G $>C$ and g.75T $>C$ mutations enhanced the activity of the SLC1 lAl promoter 1.4 (SD 0.14)-fold and $1.6(0.25)$-fold, respectively, compared with the wild type sequence. The increase in activity for the g.75T $>C$ mutation was significant $(p<0.05)$, whereas the increase in activity for the g.43G $>C$ mutation was not. The g.332C $>\mathrm{T}$ polymorphism also enhanced significantly the activity of the SLCIIAl promoter 1.6 (0.19)-fold $(\mathrm{p}<0.05)$, whereas the g.561G $>$ A mutation 


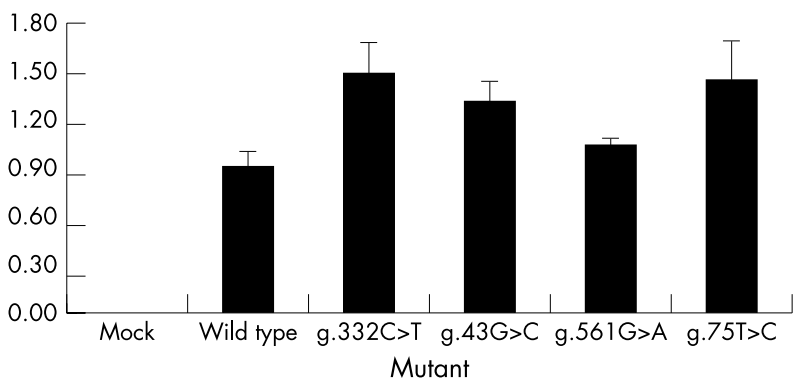

Figure 2 Mean (SD) transactivation activity of SLC11A1 promoter mutants in 293 cells. Activity of the wild type and mutant SLC1 1A1 promoters was analysed by luciferase assays. Values are expressed as luciferase activity relative to Renilla luciferase. Means of results from three independent experiments are given.

had no effect on promoter activity compared with the wild type sequence (fig 2).

\section{DISCUSSION}

The z-DNA forming polymorphic repeat in the SLC11AI promoter acts as a functional polymorphism and influences gene expression, with allele 3 having the strongest promoter activity. ${ }^{4}$ Allele 3 has been associated with hyperactivation of macrophages and may be functionally linked to susceptibility to HIV-1. ${ }^{11}$ In this study, although we did find a possible association with the presence of allele 2 and delayed disease progression, numbers were small, and this observation was not significant $(p=0.2045)$. In contrast with the literature, no associations were found with respect to the repeat polymorphism and risk of HIV infection within our population. In addition, the previously reported g.332C $>$ T single nucleotide polymorphism was not associated with susceptibility to HIV. We used a comprehensive approach to screen the promoter region of SLCIIAI and identified three novel sequence variants in combination with allele 3 of the polymorphic repeat. Although the g.75T $>$ C mutation showed an increase in promoter activity, it was not associated directly with the introduction of a transcription factor binding site or with susceptibility to HIV-1; it occurred in $1 / 8$ HIV-1 seronegative Asian participants. The lack of HIV-l seropositive Asians in this study, however, warrants further investigation of this mutation in the Asian population. The g.43G $>$ C novel mutation, which presented in 2/64 HIV-1 seropositive Africans but was absent in the 63 uninfected Africans, was shown to increase the promoter activity of SLC11A1, although this increase was not significant. The possibility that this functional mutation, which creates an additional transcription factor binding site, may lead to enhanced susceptibility to pathogen infection within the African population needs further investigation. As the exact mechanism of SLCIIAl function in HIV-l infection is unknown at present, the identification of novel population specific variants in the promoter region of this gene, which affects protein expression, may play a pivotal role in predicting susceptibility to HIV-1, particularly in populations from HIV stricken sub-Saharan Africa.

\section{ACKNOWLEDGEMENTS}

We thank Ms A Laten (Department of Medical Virology, University of Stellenbosch) for help with DNA extractions and sequencing, Ms E Carr (Cancer Research Programme, Garvan Institute of Medical Research) for help with sequencing, Dr L Breytenbach (Department of Medical Virology, University of Stellenbosch) for sample collection and maintenance of the HIV database, and all clinicians and nursing staff at the HIV clinics and blood transfusion services of the Western Cape, as well as all study participants.

\section{Authors' affiliations}

H Donninger, Departments of Internal Medicine, University of Stellenbosch, Tygerberg, South Africa

T J Cashmore, T Scriba, D C Petersen, E Janse van Rensburg, V M Hayes,

Departments of Medical Virology, University of Stellenbosch, Tygerberg, South Africa

T J Cashmore, Departments of Human Genetics, University of Stellenbosch, Tygerberg, South Africa

V M Hayes, Cancer Research Program, Garvan Institute of Medical

Research, St Vincent's Hospital, Sydney, Australia

Correspondence to: DrV M Hayes, Cancer Research Program, Garvan Institute of Medical Research, 384 Victoria Street, Darlinghurst, NSW 2010, Australia; v.hayes@garvan.org.au

Conflicts of interest: none declared.

Funding: The study was funded by the Poliomyelitis Research Foundation, South Africa.

Received: 10 August 2003

Accepted: 2 November 2003

\section{REFERENCES}

1 Blackwell JM, Goswami T, Evans CAW, Sibthorpe D, Papo N, White JK, Searle S, Miller EN, Peacock CS, Mohammed H, Ibrahim M. SLC1 1 Al (formerly NRAMP1) and disease resistance. Cell Microbiol 2001;3:773-84.

2 Ouchi K, Suzuki Y, Shirakawa T, Kishi F. Polymorphism of the SC11A1 (formerly NRAMP1) gene confers susceptibility to Kawasaki disease. JID 2003;187:326-9.

3 Blackwell JM, Barton CH, White JK, Searle S, Baker AM, Williams H, Shaw M-A. Genomic organization and sequence of the human NRAMP gene: identification and mapping of a promoter region polymorphism. Mol Med 1995; 1:194-205.

4 Searle S, Blackwell JM. Evidence for a functional repeat polymorphism in the promoter of the human NRAMP1 gene that correlates with autoimmune versus infectious disease susceptibility. J Med Genet 1999;36:295-9.

5 Graham AM, Dollinger MM, Howie SEM, Harrison DJ. Identification of novel alleles at a polymorphic microsatellite repeat region in the human NRAMP1 gene promoter: analysis of allele frequencies in primary biliary cirrhosis. J Med Genet 2000;37:150-2.

6 Marquet S, Sanchez FO, Arias M, Rodriguez J, Paris SC, Skamene E, Schurr E, Garcia LF. Variants of the human NRAMP1 gene and altered human immunodeficiency virus infection susceptibility. JID 1999;180:1521-5.

7 Blackwell JM, Searle S, Mohamed H, White JK. Divalent cation transport and susceptibility to infectious and autoimmune disease: continuation of the lty/ Lsh/Bcg/Nrampl/Slcl lAl gene story. Immunol Lett 2003;85:197-203.

8 Hayes VM. Genetic diversity of the alpha-1-antitrypsin gene in Africans identified using a novel genotyping assay. Hum Mutat 2003;22:59-66.

9 Wu Y, Hayes VM, Osinga J, Mulder IM, Looman MWG, Buys CHCM, Hofstra RMW. Improvement of fragment and primer selection for mutation detection by denaturing gradient gel electrophoresis. Nucleic Acid Res 1998;26:5432-40.

10 Lewis LA, Victor TC, van Helden EG, Blackwell JM, da Silva-Tatley F, Tullett S, Ethlers M, Beyers N, Donald PR, van Helden PD. Identification of C to T mutation at position $-236 \mathrm{bp}$ in the human NRAMP1 gene promoter. Immunogenet 1996:44:309-11.

11 Shaw MA, Clayton D, Atkinson SE, Williams H, Miller N, Sibthorpe D, Blackwell JM. Linkage of rheumatoid arthritis to the candidate gene NRAMP1 on 2q35. J Med Genet 1996;33:672-7. 